\begin{tabular}{|c|l|}
\hline Title & $\begin{array}{l}\text { Digital confocal microscopy using a virtual 4f-system based on numerical beam propagation for depth measurement } \\
\text { without mechanical scanning }\end{array}$ \\
\hline Author(s) & Goto, Y uta; Okamoto, A tsushi; Toda, Masataka; Kuno, Y asuy uki; Nozawa, Jin; Ogawa, Kazuhisa; Tomita, A kihisa \\
\hline Citation & $\begin{array}{l}\text { Japanese Journal of A pplied Physics (JJA P), 55(8), 08RE04 } \\
\text { https://doi.org/_0.7567/JJAP.55.08REO4 }\end{array}$ \\
\hline Issue Date & 2016-08 \\
\hline Doc URL & http://hdl.handle.net/2115/66896 \\
\hline Rights & ○ 2016 The Japan Society of A pplied Physics \\
\hline Type & article (author version) \\
\hline File Information & JJAP_MC15014_Manuscript.pdf \\
\hline
\end{tabular}

Instructions for use 


\section{Digital Confocal Microscopy using a Virtual 4f-System based on Numerical Beam Propagation for Depth Measurement without Mechanical Scanning}

Yuta Goto ${ }^{1 *}$, Atsushi Okamoto ${ }^{1}$, Masataka Toda ${ }^{2}$, Yasuyuki Kuno ${ }^{2}$, Jin Nozawa ${ }^{1}$, Kazuhisa Ogawa ${ }^{1}$, and Akihisa Tomita ${ }^{1}$

${ }^{1}$ Graduate School of Information Science and Technology, Hokkaido University, N14-W9, Kita-Ku, Sapporo, Hokkaido 060-0814, Japan

${ }^{2}$ Second Production Engineering Development Dept., Aisin Seiki Co., Ltd. 2-1, Asahi-machi, Kariya, Aichi, 446-8524, Japan

E-mail: goto@optnat.ist.hokudai.ac.jp

We propose a digital confocal microscope using a virtual $4 f$-system based on numerical beam propagation for depth measurement without mechanical scanning. In our technique, the information in the sample target along the depth direction is obtained by defocusing the virtual $4 f$-system, which consists of two virtual lenses arranged in a computer simulation. The principle of our technique is completely different from that of the mechanical scanning method used in the conventional confocal microscope based on digital holography. By using the virtual $4 f$-system, the measurement and exposure time can be drastically reduced because multilayered tomographic images are generated using a single measurement. In this paper, we tested the virtual depth imaging technique by measuring cover glasses arranged along the depth direction. 


\section{Introduction}

A confocal microscope ${ }^{1-5)}$ provides a background-noise-free image via a pinhole located at the focal position. Furthermore, the confocal microscope enables us to implement tomographic imaging or three-dimensional (3D) measurements, ${ }^{6,7)}$ because it can yield a depth resolution that is non-existent in conventional optical microscopes. For these advantages, the confocal microscope is commonly used in many fields, such as for the measurement of the structures of industrial products or biological samples. Furthermore, this is also expected to be applicable for in vivo imaging. ${ }^{8-10)}$

Nevertheless, obtaining a 3D image using a confocal microscope is very time-consuming, because of the 3D mechanical scanning. To exclude scanning along the transverse direction (the $x$ - and $y$-axes), confocal microscopes combined with a spin disk $\left(\right.$ Nipkow disk) ${ }^{11-13)}$ and with a paired microlens array and pinhole array ${ }^{14-16)}$ have been proposed. These methods can potentially allow simultaneously measurements without mechanical scanning on the $x y$-plane. On the other hand, methods using electrical and acousto-optical focus-tunable lens have also been proposed ${ }^{17-19)}$ for scanning along the depth direction (z-axis) with high speed. However, if the abovementioned techniques are used, then depth scanning is required in the actual optical system. This increases the acquisition time required for images and the exposure time for the target object. As a result, the quality of the image reconstruction is deteriorated as a result of imaging artifacts from the movement of the target sample. Moreover, in the case of biological imaging, the long exposure time for the target object may cause problems such as damage to biological tissues and fluorescence fading.

Meanwhile, in conventional confocal microscopy, digital processing is required in order to reproduce the $3 \mathrm{D}$ image from the intensity information of each position. ${ }^{20)}$ In addition, with the rapid development of computer hardware, several methods using digital holography $(\mathrm{DH})^{21-25)}$ for confocal microscopy have been proposed. The resulting devices are known in the literature as digital confocal microscopes. It has been reported that the quality of reconstructed images can be improved by simulating the beam propagation in a confocal configuration and dynamically locating a virtual pinhole ${ }^{26)}$. The aberration compensation of the objective lens uses a virtual offset lens, ${ }^{27)}$ and confocal phase image measurements combining the confocal microscope and $\mathrm{DH}$ have also been reported. ${ }^{28,29)}$ In addition, the digital confocal microscope has been optimized for fluorescence imaging using Fresnel incoherent holography. ${ }^{30)}$ Although the aforementioned methods for digital confocal microscopy ${ }^{20,26-30)}$ enable one to improve or extend the power of the confocal microscope, 
mechanical scanning along the depth direction is still required because those methods utilize only the complex amplitude at the focal plane of the objective lens.

To realize a depth measurement without mechanical scanning, we propose a digital confocal microscope using a virtual $4 f$-system based on numerical beam propagation. In our technique, all complex amplitudes of the multiple scattered beams are captured using $\mathrm{DH}$. Next, numerical beam propagation is implemented using the captured complex-amplitude image in a computer simulation by following three computational steps: the image is processed by a $4 f$-system (referred to as a virtual 4 -system), then, by a fast Fourier transform (FFT), and finally, the image is processed by a virtual pinhole aperture. Within these three steps, the FFT and multiplication by the pinhole aperture can be performed in the same manner as that with conventional digital confocal microscopy. ${ }^{26)}$ In our method, by defocusing one of the virtual lenses in the virtual $4 f$-system, one can compensate for a spherical wavefront in the multiple scattered beams outside the focal plane of the objective lens. Thereby, the component of the depth position, depending on the extent of defocus in a virtual lens, can be extracted from all the multiple scattered beams. Therefore, by iteratively processing the initial image using the virtual $4 f$-system with varying degrees of focus, depth information can be acquired from a single captured complex amplitude without mechanical scanning. Consequently, this technique dramatically improves the measurement speed of a digital confocal microscope, without mechanical scanning, and without using additional optical elements.

In this paper, we first describe the basic operation of the virtual optical system for a confocal microscope and the simplification of its calculation in Sections 2.1 and 2.2. Next, after verifying this technique experimentally in Sections 3.1 and 3.2, we evaluate and discuss the depth resolution in Section 3.3. Finally, in Section 3.4, we demonstrate the depth imaging of a cover glass without mechanical or electronic scanning in an actual optical system by using this technique.

\section{Method}

\subsection{Basic operation}

In this section, we describe the basic operation of our technique. Our technique can be divided into two domains: the actual optics domain for measuring the target sample, as shown in Fig. 1(a), and the virtual optics domain for controlling the depth-measurement position and filtering undesired noise, as shown in Fig. 1(b). In our technique, the whole of the virtual optics domain is performed in a computer via $\mathrm{DH}$. 
In the actual optics domain, the collimated beam from the laser source is focused onto a measurement target via the objective lens. This beam is then reflected (back-scattered) from the target sample and is referred to as the "scattered signal beam". Here, the target sample is assumed to be a multi-layered object in order to simplify the explanation of the basic operations. Thus, the beam from the target sample can be treated as a multiple-scattered beam reflecting from those multiple layers. Afterward, the complex amplitude of the scattered signal beam is captured by the $\mathrm{DH}$.

In the virtual optics domain, two virtual lenses arranged to constitute the so-called "4f-system" are applied to the scattered signal beam captured in the actual optical domain. In our technique, these two virtual lenses are referred to as the "virtual $4 f$-system”. Here, we describe the protocol for the calculation of the virtual 4 -system. First, the function of free-space propagation between the input plane and the first lens plane is multiplied by the original (captured) complex-amplitude distribution, and then the phase factor of the lens, $\exp \left[i k\left(d x^{2}+d y^{2}\right) / f\right]$, is multiplied by the propagated complex-amplitude distribution. Here, $k$ is the wavenumber, $f$ is the focal length of both of the virtual lenses, and $d x$ and $d y$ are the positions along the $x$ - and $y$-axes, respectively. Next, the resultant complex-amplitude distribution is multiplied by the function of free-space propagation between the first lens plane and the second lens plane. Finally, after multiplying the propagated complex-amplitude distribution by the above mentioned phase factor of the lens again, the free-space propagation between the second lens plane and the imaging plane is calculated. In the case that the measurement plane is shifted by a depth $\Delta z$, the virtual lens needs to be defocused by a distance $-2 \Delta z$. This is done by varying the propagation distance between the first lens plane and the second lens plane in the virtual $4 f$-system. (Note that, considering the reflection of the beam in the target sample, the actual amount of displacement in measurement plane becomes $2 \Delta \mathrm{z}$.) In other words, the amount of defocus (the shifted distance) of the lens in the virtual $4 f$-system determines the measurement position along the $z$-axis.

After implementing the virtual $4 f$-system, the complex-amplitude distribution of the focal plane is calculated using the FFT method, and is multiplied by the pinhole pattern:

$$
\text { Pinhole }=\left\{\begin{array}{ll}
1 & \left(\sqrt{x^{2}+y^{2}} \leq D\right) \\
0 & \left(\sqrt{x^{2}+y^{2}}>D\right)
\end{array},\right.
$$

where $D$ is the pinhole radius. Thus, this technique also filters out the background noise from the non-measurement position in the same way as in the conventional confocal 
microscope. In addition, all of the data along the depth direction can be acquired through iterative calculation using the abovementioned protocol for the calculation of the virtual $4 f$-system while continuously shifting the lens position.

By carrying out the above operations, our technique can measure the tomographic data along the $z$-axis without mechanical scanning. In addition, by using our technique, the configuration of a confocal microscope is simplified by replacing a certain component of the actual optical system with a virtual optical system.

\subsection{High-speed calculation of a virtual $4 f$-system using a defocus wavefront}

In the above calculation of the virtual $4 f$-system, the free-space propagation function of the beam is essential. In general, the calculation of the free-space propagation function includes a convolution integral, which is extremely time-consuming. Even if the FFT is used, which can be calculated faster than the convolution integral, the full faster calculation is difficult because the virtual $4 f$-systemrequires multiple free-space propagation functions. In addition, the phase factor of the lens is used to implement the virtual lens, but in the case of a lens with a high numerical aperture (NA), the large curvature of its phase factor causes calculation errors as a result of aliasing.

To solve these problems, we introduce a method for calculation using the defocus wavefront for the virtual $4 f$-system. In a typical $4 f$-system, when defocusing of the lens, the relayed image plane of that system to have a spherical phase-distribution. This spherical phase-distribution is referred to as the "defocus wavefront”, $\phi_{\text {def }}$, and the function of the complex amplitude including $\phi_{\text {def }}$ can be calculated as exp $\left[i k \Delta d\left(x^{2}+y^{2}\right) / f^{2}\right] .{ }^{31)}$ Here, $\Delta d$ is the amount of defocus (the shift distance of lens). Therefore, this function of the complex amplitude provides the same effect as a virtual $4 f$-system. The calculation of the defocus wavefront is faster than that using the virtual $4 f$-system because it does not require a convolution integral or the FFT. The computational complexity of the FFT and that of the defocus wavefront are given by $\mathrm{O}\left(N^{2} \log N^{2}\right)$ and $\mathrm{O}\left(N^{2}\right)$, respectively. Thus, the computational complexity of calculating the defocus wavefront $\phi_{\text {def }}$ is less than that for the FFT. In addition, the curvature of the phase factor of the lens and the defocus wavefront $\phi_{\text {def }}$ are defined as $1 / f$ and $\Delta d / f^{2}$, respectively. Therefore, the curvature of the defocus wavefront $\phi_{\text {def }}$ is less than that of the phase factor of the lens. Thus, this technique can simultaneously reduce the calculation time and the aliasing error. 


\section{Experiment}

\subsection{Setup}

We performed an experiment to verify the principles of our technique. The experimental setup is shown in Fig. 2. Firstly, the beam from the laser, whose wavelength $\lambda$ is $532 \mathrm{~nm}$, is focused by OL1 and passed through the pinhole. The beam is then collimated by L3, and radiated to the phase-type spatial light modulator (PSLM), which has a pixel size of $20 \times 20$ $\mu \mathrm{m}^{2}$ and a pixel number of $800 \times 600$, and the gray-level for the $2 \pi$ modulation is 157 . The beam modulated by the PSLM is passed through relay lenses (L1, L2), and then that beam is focused by lens OL2 with an NA of 0.42 .

In this experiment, the PSLM is used for scanning the probe beam along the $x$-axis and $y$-axis directions on the focal plane. After focusing the probe beam with OL2, the position of the focal point is shifted by $\Delta x$ and $\Delta y$ along the $x$-axis and $y$-axis directions, respectively. Indeed, the optical axis of the probe beam can be inclined along the $x$-axis and $y$-axis at angles of $\theta_{x}=\tan ^{-1}\left(\Delta x / f_{\mathrm{OL}}\right)$ and $\theta_{y}=\tan ^{-1}\left(\Delta y / f_{\mathrm{OL}}\right)$, respectively, by displaying the phase distribution $\phi(x, y)=-i k\left\{\sin \theta_{x} x+\sin \theta_{y} y\right\}$ on the PSLM. Here, $x$ and $y$ are the positions of the $x$ - and $y$-coordinates on the PSLM, respectively, and $f_{\mathrm{OL}}$ is the focal length of the OL2 lens.

Next, the probe beam is reflected from the target sample as the scattered signal beam, and its complex-amplitude distribution is detected using holographic diversity interferometry (HDI), ${ }^{32,33)}$ a DH method with high spatial resolution. The CCD that comprises the HDI has a pixel size of $3.75 \times 3.75 \mu \mathrm{m}^{2}$ and a pixel number of $1280 \times 960$.

Figure 3(a) shows the phase image displayed on the PSLM at $\Delta x=27.66 \mu \mathrm{m}$ and $\Delta y=$ $0.00 \mu \mathrm{m}$, and Fig. 3(b) shows the phase image captured using HDI at that time. This experiment simplifies scanning on the $x y$-plane in order to verify the basic operation of the virtual $4 f$-system, by eliminating scanning along the $y$-axis. In the computer, the captured complex-amplitude distribution of the scattered signal beam is reduced by half after trimming it to the image size of $800 \times 800$ pixels in order to compensate for the difference in pixel size between the PSLM and the CCD. Afterward, the phase distribution $-\phi(x, y)$ is multiplied by the complex-amplitude distribution of the scattered signal beam, in order to cancel out the phase distribution produced by the PSLM. Finally, the reconstructed image is obtained by performing virtual optics.

\subsection{Verification of the basic operation of the virtual $4 f$-system}

We verified the basic operation of the virtual $4 f$-system by comparing the 
complex-amplitude distribution before and after processing of the virtual $4 f$-system when the defocused mirror was measured. In this part, the amount of defocus of the mirror was set to $100.0 \mu \mathrm{m}$. Figure 4 shows a phase image and phase profile captured using HDI when the mirror defocused at $100.0 \mu \mathrm{m}$ was measuring. By defocusing the mirror, a spherical wavefront appeared, as shown in Figs. 4(a) and 4(d). Moreover, Figs. 4(b) and 4(e) show the phase image and phase profile of the defocus wavefront synthesized by the virtual $4 f$-system. Then, by multiplying the synthesized defocus wavefront and cancelling out the defocus of the mirror, the captured phase image was modulated into the plane wave, as shown in Figs. 4(c) and 4(f). Indeed, the plane wave shown in Figs. 4(c) and 4(f) was recovered from the captured phase image shown in Figs. 4(a) and 4(d), although the wavefront was deteriorated because of shot-noise in the image sensor, insufficient alignment, and aberrations in the actual optical-system. This means that, by using the virtual $4 f$-system, the measurement position can be shifted along the $z$-axis.

In addition, after capturing the reflected beam from the mirror defocused at $100.00 \mu \mathrm{m}$, the operation of the virtual optical system including the virtual $4 f$-system was iteratively calculated while continuously shifting the position of the virtual lens. As the result, the depth information can be reconstructed, as shown in Fig. 5. The reconstructed intensity image on the $x z$-plane and the reconstructed intensity profile at $x=3.20 \mu \mathrm{m}$ are shown in Figs. 5(a) and 5(b), respectively. Here, this reconstructed intensity image is sliced from the volume data, which is composed of multiple transverse-section images of each depth position produced using the virtual 4f-system. In Fig. 5(a), the undesired intensity peak appears in the center of the reconstructed intensity image because stray light, which is not inclined to the axial angle by the PSLM, becomes mixed in the scattered signal beam. According to Figs. 5(a) and 5(b), the depth position of the intensity peak is located at 95.85 $\mu \mathrm{m}$. Although there is an error of approximately $4 \%$ in the measured depth position due to the aberration of the actual optical system and the imperfect position accuracy in the mirror placement, this measured depth position is in good agreement with that of the defocused mirror.

Therefore, these results show that our technique can measure depth information about a target object without mechanical scanning. Nevertheless, in Fig. 5(a), the intensity distribution was decreased in accordance with the deviation along the $x$-axis from the center. This is because the focal position of the signal beam after the FFT was deviated from the position of the virtual pinhole by the aberration in the actual optical system. However, dynamically arranging the pinhole ${ }^{26)}$ in accordance with the deviation of the 
focal position of the signal beam enables us to solve this problem.

\subsection{Evaluation of the depth resolution}

Here, we discuss the depth resolution of our technique. The depth resolution is evaluated by measuring the depth position of a mirror arranged at the focal plane of the OL2 lens, as shown in Fig.2. Here, the depth scanning was performed by defocusing the virtual $4 f$-system without actual scanning of the target sample. In addition, the size of the virtual (pixelated) pinhole is defined using the Nyquist aperture like so:

$$
D_{p}=\frac{N x \Delta x}{\lambda f} D
$$

where $D_{p}$ is the pixelated pinhole size, $N_{X}$ is the grid number of the calculation space for the virtual optical system, $\Delta x$ is the grid pitch of the calculation space for the virtual optical system, $f$ is the focal length of the correction lens implemented by the FFT, and $D$ is the pinhole size before pixelation. In general, the optimized pinhole size, which is balanced between the transmitted intensity and the depth resolution, is determined by the diameter of the Airy disk, which is defined as $1.22 \lambda / \mathrm{NA}$, and its pinhole size is normalized to " 1 airy unit (AU)”.

Figure 6 shows the plotted normalized intensity of each defocus position of the virtual $4 f$-system as an optical sectioning $(I-z)$ curve. According to Fig. 6, the peak of the intensity corresponds to the position of the mirror, $z=0.00 \mu \mathrm{m}$, and the intensity plummets with the defocusing of the virtual $4 f$-system. Therefore, we verified that the background noise from the non-measurement position was filtered out using our technique, and its full-width half-maximum (FWHM) at $1.19 \mathrm{AU}$ was $10.77 \mu \mathrm{m}$. Furthermore, when the size of the virtual pinhole was reduced to $1 \times 1$ pixels (0.39 AU), the FWHM improved to $5.31 \mu \mathrm{m}$. This result suggests that the depth resolution of our technique can be improved by reducing the size of the virtual pinhole. In addition, the signal beam passed through the pinhole in the virtual optical system, not in the actual optical system. It is therefore possible to prevent the deterioration of the signal intensity caused by decreasing the pinhole diameter.

\subsection{Depth imaging of a cover glass}

In the following, using our technique, we demonstrate the simultaneous depth imaging of a cover glass along the $z$-axis. Here, the cover glass, which has thickness specifications of $150.00 \pm 20.00 \mu \mathrm{m}$ and a refractive index of 1.53 , was employed as a measurement target, 
and the center of this cover glass was located at $z=0.00 \mu \mathrm{m}$. The cover glass was affected by refraction because it was located at the focal position. Thus, the apparent thickness of the cover glass was approximately $100.00 \mu \mathrm{m}$. Figure 7(a) shows the sliced image on the $x z$-plane from volume data, which is composed of multiple transverse images of each depth position produced using the virtual $4 f$-system, and Fig. 7 (b) shows the intensity profile at $y$ $=0.00 \mathrm{~mm}$. According to Figs. 7(a) and 7(b), the first and second peaks of the reconstructed intensity correspond to the positions of the front and back surfaces on the cover glass, respectively. The intensity peak corresponding to the back surface of the glass is reduced because the light radiating to the back surface is reduced due to partial reflection at the front surface. In addition, the distance between the two peaks is $100.16 \mu \mathrm{m}$, which is in good agreement with the thickness of the cover glass.

In addition, we show the reconstructed depth information from measuring two cover glasses stacked along the optical axis. Likewise, according to Fig. 8, the three intensity peaks correspond to the positions of the reflection surfaces of the cover glass. Comparing the intensity peaks at $-100 \mu \mathrm{m}$ and $100 \mu \mathrm{m}$, the intensity peak at $100 \mu \mathrm{m}$ is smaller than that of the $-100 \mu \mathrm{m}$, again because of partial reflection (the same reason as in Figs. 7(a) and 7(b)). Moreover, the intensity peaks at $-100 \mu \mathrm{m}$ and $100 \mu \mathrm{m}$ are smaller than the peak at $0 \mu \mathrm{m}$. This is because the beam is spread due to the defocused depth position of the measurement plane, and the intensity of the beam transmitted through the pinhole is decreased. In addition, the intensity distribution shown in Fig. 8(a) is decreased in accordance with the deviation along the $x$-axis from the center, similar to the case in Fig. 5(a). This can also be solved by dynamically arranging the pinhole.

Therefore, these results showed that our technique can obtain tomographic images without mechanical scanning.

\section{Conclusions}

We proposed a digital confocal microscope using a virtual $4 f$-system based on numerical beam propagation for depth measurement without mechanical scanning. We verified the basic operation of our technique and evaluated its depth resolution. In addition, we demonstrated simultaneous depth imaging of cover glasses. The experimental results showed that our technique can collectively measure the depth information of cover glasses used as target objects, using a single complex-amplitude distribution of a captured multiple-scattered beam without mechanical scanning. This technique has great potential to improve the measurement speed of the confocal microscope. 


\section{References}

1) M. Minsky, U.S. Patent 3013467 (1961).

2) T. R. Corle, C.-H. Chou, and G. S. Kino, Opt. Lett. 11, 770 (1986).

3) D. M. Shotton, J. Cell Sci. 94, 175 (1989).

4) A. F. Gmitro and D. Aziz, Opt. Lett. 18, 565 (1993).

5) S. W. Paddock, Bio Techniques 27, 992 (1999).

6) K. Carlsson, P. E. Danielsson, R. Lenz, A. Liljeborg, L. Majlbf, and N. Aslund, Opt. Lett. 10, 53 (1985).

7) C. J. R. Sheppard, Min Gu and X.Q. Mao, Opt. Commun. 81, 281 (1991).

8) M. Rajadhyaksha, M. Grossman, D. Esterowitz, R. H. Webb, and R. R. Anderson, J. Invest. Dermatol104, 946 (1995).

9) B. R. Masters, and P. T. C. So, Opt. Express 8, 2 (2001).

10) Y. Hwang, J. Ahn, J. Mun, S. Bae, Y. U. Jeong, N. A.Vinokurov, and P. Kim, Opt. Express 22, 11465 (2014).

11) G. Q. Xiao, and G. S. Kino, Proc. SPIE 0809, 107 (1987).

12) J. Conchello and J. W. Lichtman, Appl. Opt. 33, 585 (1994).

13) S. Stehbens, H. Pemble, L. Murrow, and T. Wittmann, Methods Enzymol. 504, 293 (2012).

14) H. J. Tiziani, and H. M. Uhde, Appl. Opt. 33, 567 (1994).

15) H. J. Tiziani, R. Achi, R. N. Krämer, and L. Wiegers, Appl. Opt. 35, 120 (1996).

16) M. Eisner, N. Lindlein, and J. Schwider, Opt. Lett. 23, 748 (1998).

17) L. Yang, A. M. Raighne, E. M. McCabe, L. A. Dunbar, and T. Scharf, Appl. Opt. 44, 5928 (2005).

18) J. M. Jabbour, B. H. Malik, C. Olsovsky, R. Cuenca, S. Cheng, J. A. Jo, Y. L. Cheng, J. M. Wright, and K. C. Maitland, Biomed. Opt. Express 19, 645 (2014).

19) M. Duocastella, G. Vicidomini, and A. Diaspro, Opt. Express 22, 19293 (2014).

20) K. Carlsson and N. Aslund, Appl. Opt. 26, 3232 (1987).

21) J. Schwider, Appl. Opt. 28, 3889 (1989).

22) I. Yamaguchi, and T. Zhang, Opt. Lett. 22, 3889 (1997).

23) T. Zhang, and I. Yamaguchi, Opt. Lett. 23, 1221 (1998).

24) T. Nomura, S. Murata, E. Nitanai, and T. Numata, Appl. Opt. 45, 4873 (2006).

25) Y. Awatsuji, T. Tahara, A. Kaneko, T. Koyama, K. Nishio, S. Ura, T. Kubota, and O. Matoba, Appl. Opt. 47, D183 (2008).

26) A. S. Goy, and D. Psaltis, Opt. Express 20, 22720 (2012). 
27) Y. Chew, M. Shiu, J. Wang, and C. Chang, Appl. Opt. 53, G184 (2014).

28) A. S. Goy, M. Unser, and D. Psaltis, Biomed. Opt. Express 4, 1091 (2013).

29) C. Liu, S. Marchesini, and M. K. Kim, Opt. Express 22, 17830 (2014).

30) R. Kelner, B. Katz, and J. Rosen, Optica 1, 70 (2014).

31) K. Okada, K. Amaya, and Y. Onishi, Jpn. J. Opt. 41, 627 (2012) [in Japanese].

32) A. Okamoto, K. Kunori, M. Takabayashi, A. Tomita, and K. Sato, Opt. Express 19, 13436 (2011).

33) J. Nozawa, A. Okamoto, A. Shibukawa, M. Takabayashi, and A. Tomita, Appl. Opt. 54, 8644 (2015). 


\section{Figure Captions}

Fig. 1. (Color Online) Schematic diagram of our technique: (a) the actual optics domain for measuring the target sample and (b) the virtual optics domain for controlling the depth-measurement position and filtering undesired noise.

Fig. 2. (Color Online) Experimental setup. L: Lens, OL: Objective Lens, M: Mirror, BS: Beam Splitter, PBS: Polarizing BS, HWP: Half-Wave Plate, QWP: Quarter-Wave Plate, PSLM: Phase-only LCoS SLM (Hamamatsu, x10468-01), CCD: Charge-Coupled Device (Allied Vision Technology, Stingray F125B).

Fig. 3. The displayed and captured phase distributions: (a) the phase image displayed on the PSLM at $\Delta x=27.66 \mu \mathrm{m}$ and $\Delta y=0.00 \mu \mathrm{m}$, and (b) the phase image captured using HDI at the same time.

Fig. 4. (Color Online) Phase images found (a) without the virtual $4 f$-system, (b) with the defocus wavefront synthesized by the virtual $4 f$-system, and (c) with the virtual $4 f$-system. Phase profiles at $y=0.00 \mu \mathrm{m}$ found (d) without the virtual 4 -system, (e) with the defocus wavefront synthesized by the virtual $4 f$-system, and (f) with the virtual $4 f$-system.

Fig. 5. (Color Online) Reconstructed depth information when measuring the mirror at $z=$ $100.00 \mu \mathrm{m}$ : (a) the sliced image (xz-plane) from volume data, which is composed of multiple transverse images at each depth position found using the virtual $4 f$-system; and (b) the intensity profile at $x=3.20 \mu \mathrm{m}$. The peak position is at $95.85 \mu \mathrm{m}$.

Fig. 6. (Color Online) Optical sectioning ( $I-z)$ curve of confocal microscope using our technique, when the pinhole size is $1.19 \mathrm{AU}$ (blue points and line) and when it is $0.39 \mathrm{AU}$ (red points and line).

Fig. 7. (Color Online) Reconstructed depth information from measuring a single cover glass: (a) the sliced image on the $x z$-plane from volume data, which is composed of 
multiple transverse images of each depth position produced using the virtual $4 f$-system, and (b) the intensity profile at $y=0.00 \mathrm{~mm}$.

Fig. 8. (Color Online) Reconstructed depth information from measuring two cover glasses stacked along the optical axis: (a) the sliced image ( $x z$-plane) from volume data, which is composed of multiple transverse images of each depth position produced using the virtual $4 f$-system, and (b) the intensity profile at $y=0.00 \mathrm{~mm}$. 


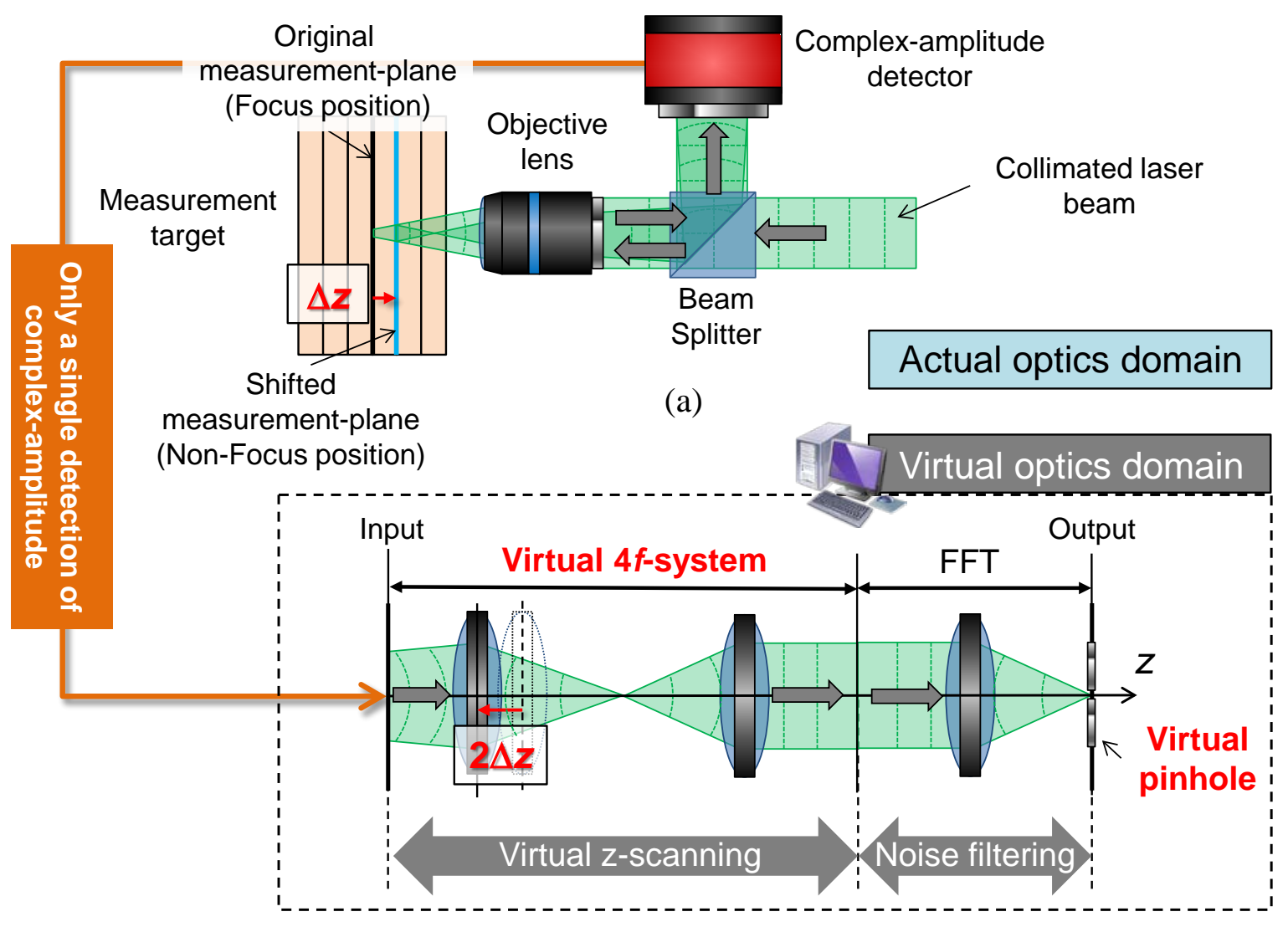

(b)

Fig. 1 (Color Online) 


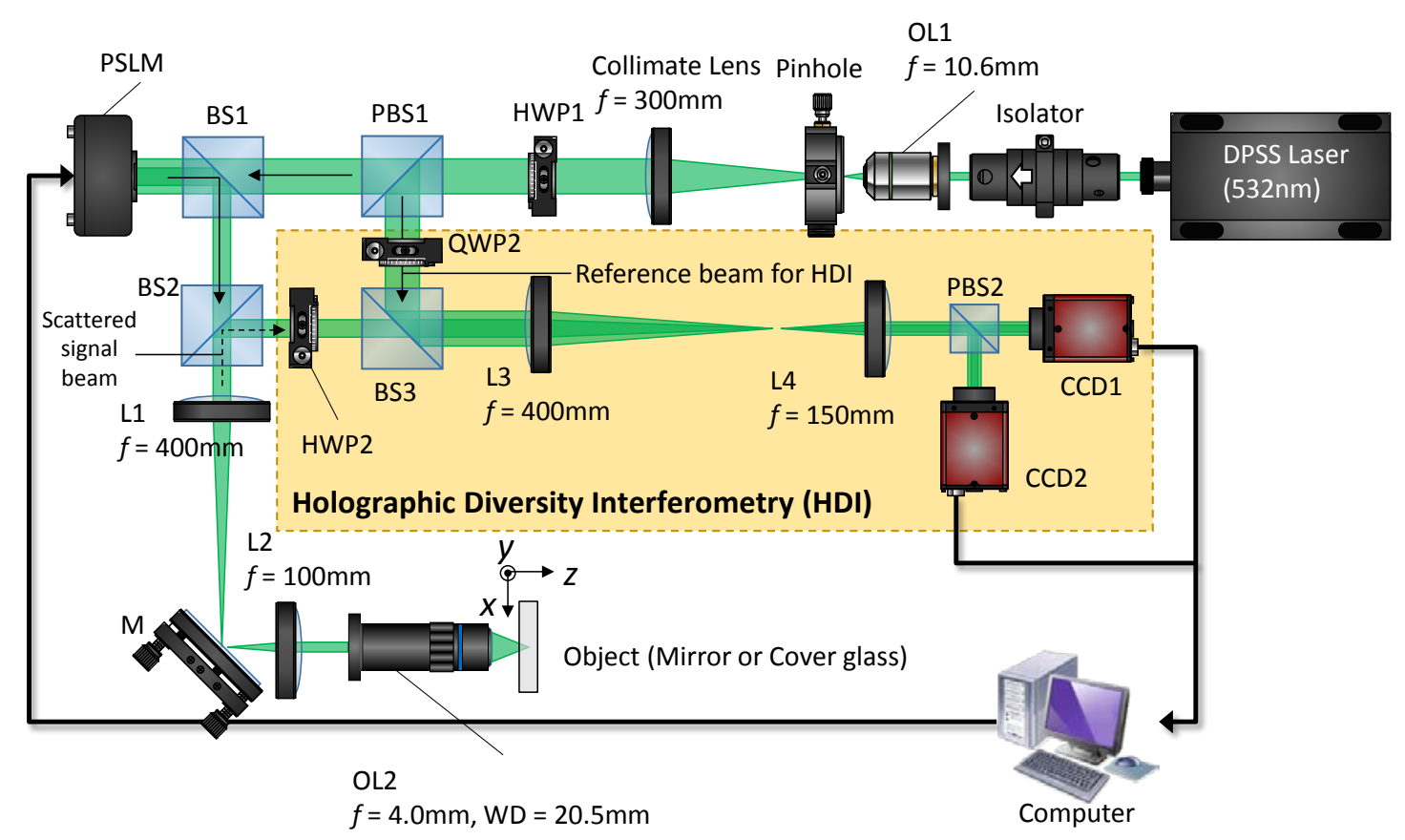

Fig. 2 (Color Online) 


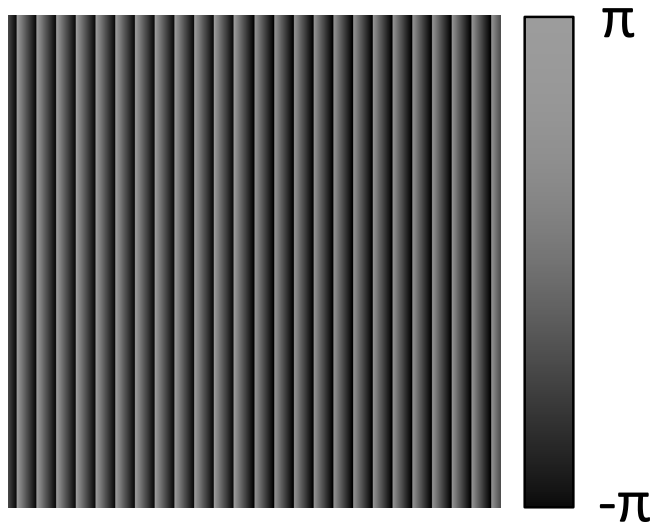

(a)

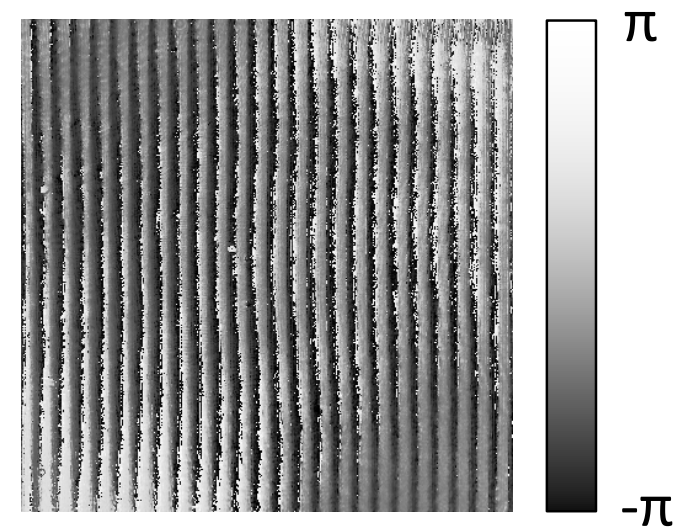

(b)

Fig. 3 


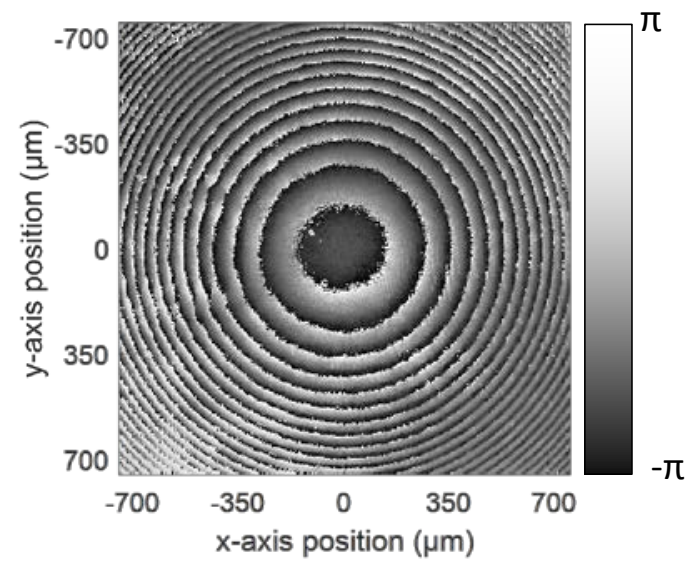

(a)

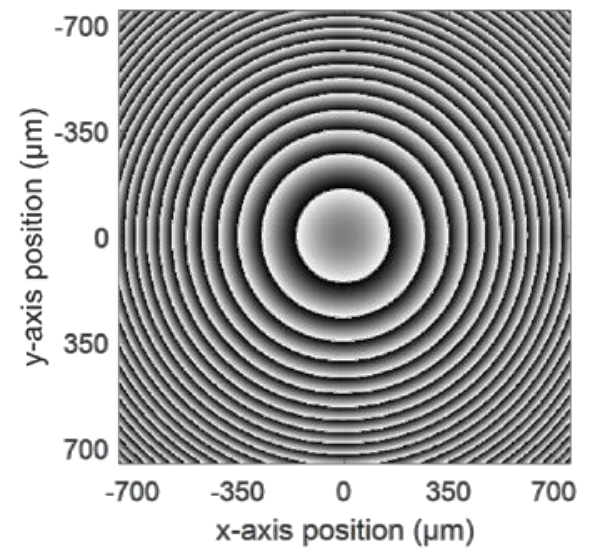

(b)

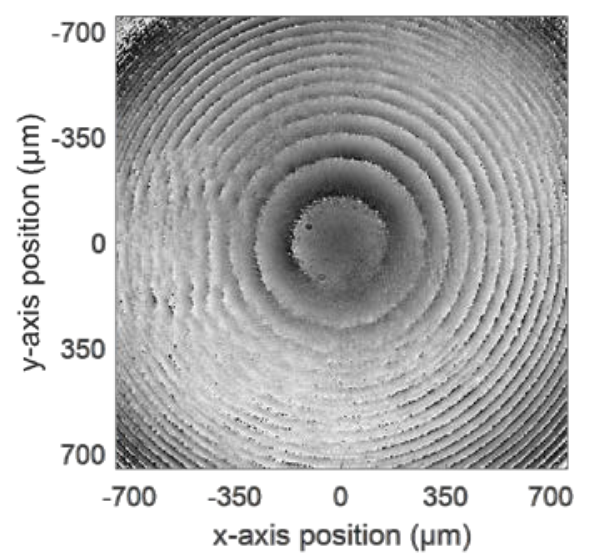

(c)

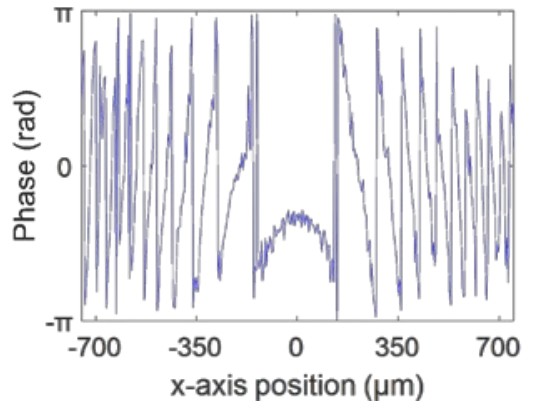

(d)

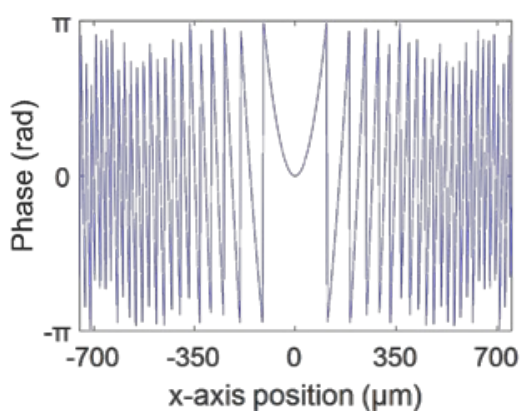

(e)

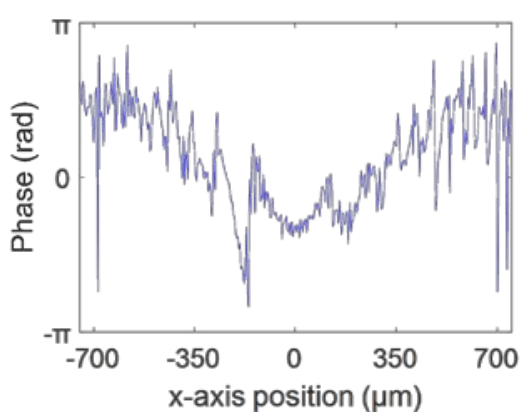

(f)

Fig. 4 (Color Online) 


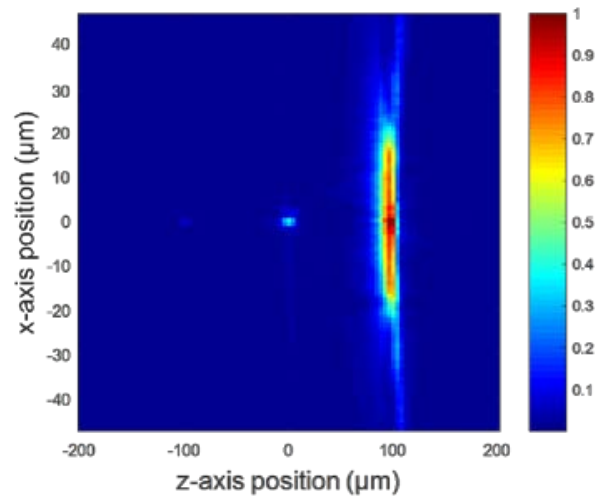

(a)

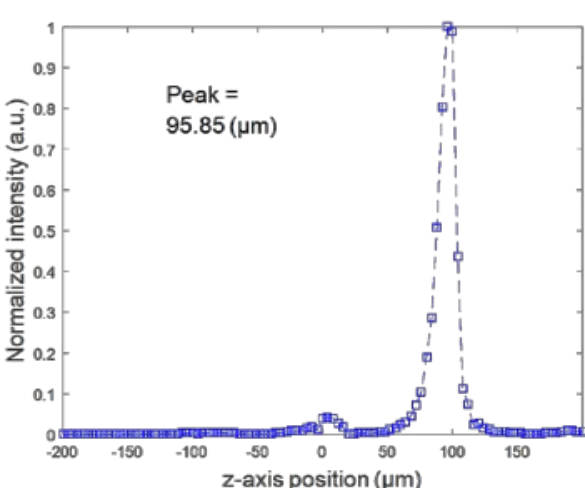

(b)

Fig. 5 (Color Online) 


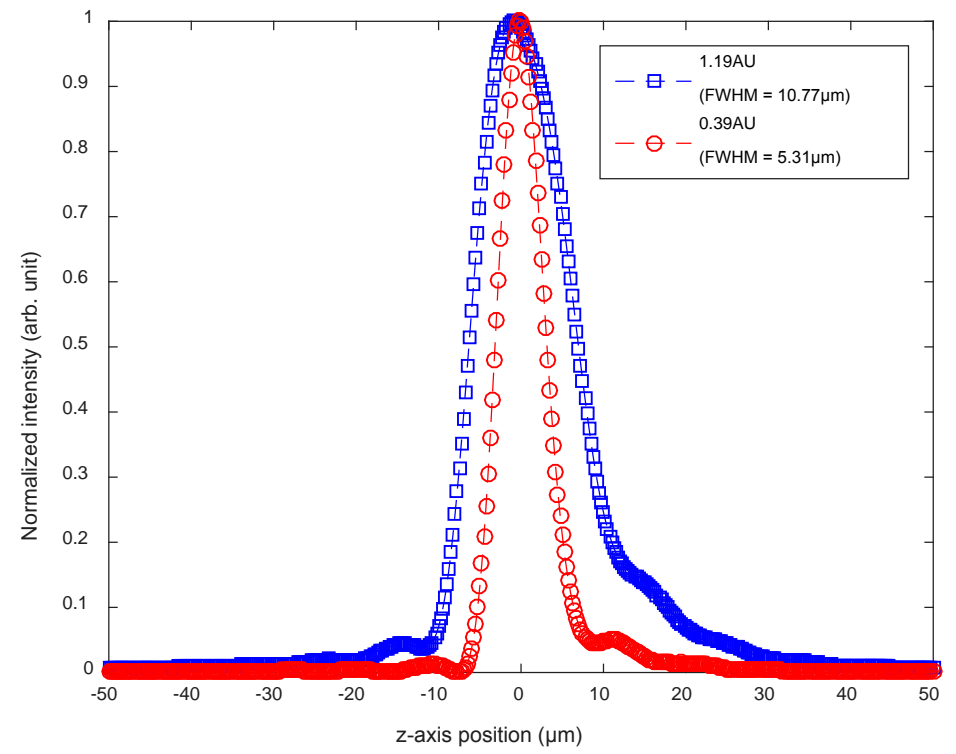

Fig. 6 (Color Online) 


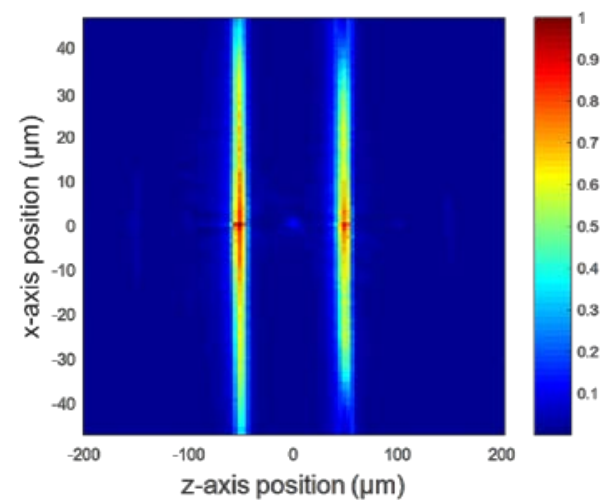

(a)

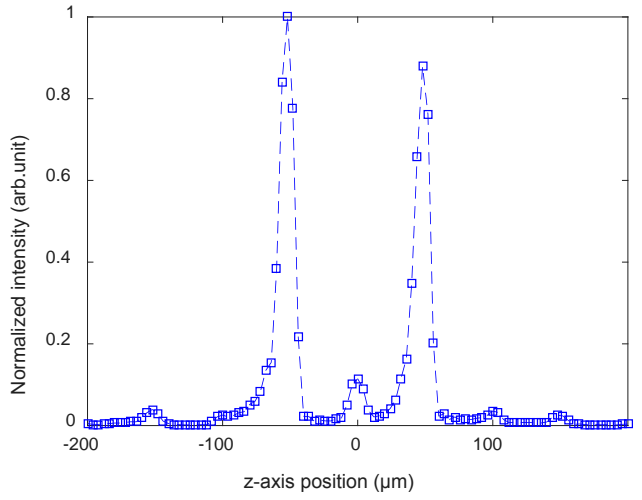

(b)

Fig. 7 (Color Online) 


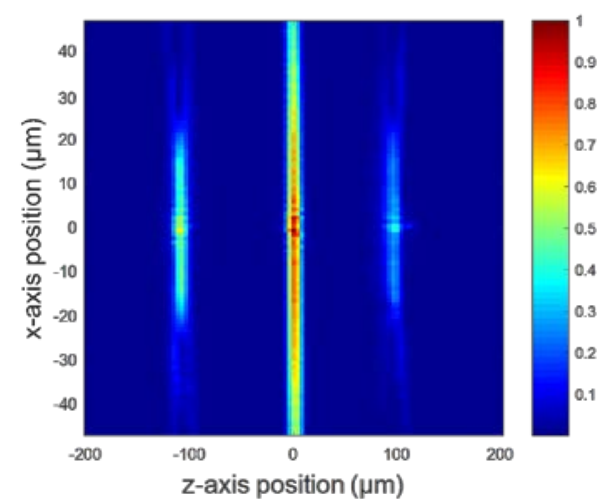

(a)

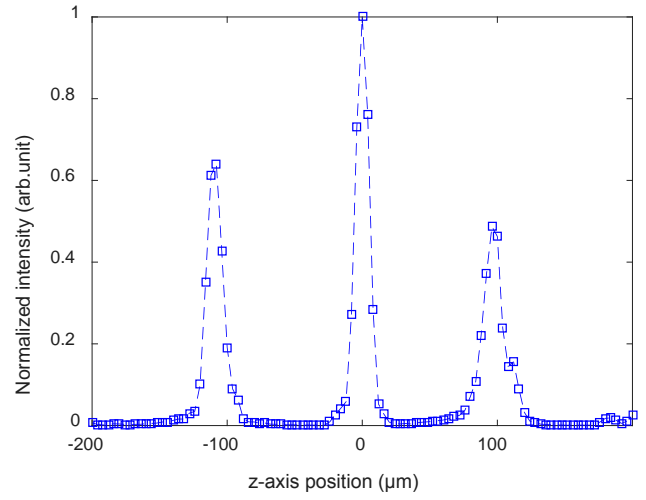

(b)

Fig. 8 (Color Online) 